\title{
VERIFICATION OF POSITIONAL ACCURACY OF ELECTRONIC PORTAL IMAGING DEVICE BY USING GRATICULE IMRT PHANTOM
}

\section{Oncology}

Chhape Ram

Asst. Prof. Medical Physicist, Swami Rama cancer Hospital and Research Institute Rampur Road Haldwani UK - 263139.

Ranjit Singh

Medical Physicist Department of Radiotherapy PGIMER-Chandigarh-160012

Balbir Singh

Medical Physicist cum RSO Department of Radiation Oncology MAX Hospital Bathinda, Punjab-151001.

Gaganpreet Singh Medical Physicist Department of Radiotherapy PGIMER -Chandigarh -160012.

Vinod Pandey*

Medical Physicist \& RSO, Swami Rama cancer Hospital and Research Institute Rampur Road Haldwani UK - 263139.*Corresponding Author

\section{ABSTRACT}

EPID (Electronic Portal Imaging Device) is ubiquitous in the radiation therapy clinic and provides a powerful and flexible tool to collect and process data in a quantitative manner to improve treatment accuracy for virtually any treatment site. This study provides an overview of the clinical implementation process for effective use of EPID. A proper control of the geometrical accuracy of treated patients during radiotherapy results in higher quality of treatment and may lead to the increase in therapeutic gain. In this study an evaluation of an electronic portal imaging was made in the following aspects: the quality of images, the estimation of prolongation of the treatment, corrections introduced after corrections.

\section{KEYWORDS}

EPID Electronic Portal Imaging Device, Clinical Implementation, Therapeutic Gain.

\section{INTRODUCTION}

Geometrical error is deviation between the intended geometry of radiotherapy plan and real geometry of radiotherapy treatment. Total geometrical errors are buildup of smaller errors, which can be generally classified as setup, organ motion and the technical condition related errors. [1]

The clear distinct must be made between the systematic and random component of these errors and its amount should be encountered in treatment planning process. Errors measuring for specific patient group with electron portal imaging device and proper correction strategy enables to predict minimize, and keep under control the amount for most of the geometrical errors, it also improves the preciseness of treatment and consequent result

Treatment verification is an important component of radiotherapy. [2] The role of verification is primarily to detect treatment delivery errors and secondly, to assess the suitability of the size of the margins planned around the clinical target volume that allows for the uncertainty in the radiotherapy process. [3] Geometric verification process must be carried out within a clearly defined structure, adhering to locally defined protocols. [4] Objective of this study is to find out the positional accuracy of EPID by using Graticule IMRT phantom. The primary purpose of port filming is to verify the treatment volume under actual conditions of treatment. Although the image quality with the megavoltage $\mathrm{x}$-ray beam is poorer than with the diagnostic or the simulator film, a port film is considered mandatory not only as a good clinical practice but as a legal record. [5,6] Verification methods begins with identifying the equipment needed for verification (for reference image acquisition, treatment image acquisition, image matching and data storage) establishing the connectivity methods between the equipment, and determining the quality levels and tests needed to maintain verification standards. [7] Earlier, port film was used to verify the patient position but now EPID used as effective technique to measure and reduce set-up errors relative to their intended set-up geometry.

\section{MATERIALSAND METHODS}

In this study IMRT Graticule phantom has been taken and anterior and lateral EPID of intended errors of $0.5 \mathrm{~cm}, 1.0 \mathrm{~cm}$ and $1.5 \mathrm{~cm}$ had been introduced at the different source to imager distance (SID) likely $\mathrm{P} 2=140 \mathrm{~cm}, \mathrm{P} 3=150 \mathrm{~cm}, \mathrm{P} 4=160 \mathrm{~cm}$ and $\mathrm{P} 5=180 \mathrm{~cm}$ respectively. In anterior EPID longitudinal and lateral shifts has been observed and in lateral EPID longitudinal, lateral and vertical shifts has been observed but the shifts in lateral direction are less than or equal to $0.04 \mathrm{~cm}$ hence can be neglected as according to our study requirements. In this study, a Medical Linear Accelerator Clinac DBx-1160 (Varian, Palo, alto, CA) from Varian medical system having single photon energy of $6 \mathrm{MV}$ is used. Linear Accelerator have inbuilt Portal vision a-Si 500 for portal imaging.

IMRT Graticule Phantom is a phantom made up of tissue equivalent material and have a dimension of $18 \mathrm{~cm} \times 18 \mathrm{~cm} \times 18 \mathrm{~cm}$. This phantom is quite useful to carry out port film dosimetry for various treatment modalities which involves non uniform dose fluence and where the dose distribution is very complex. This Phantom is provided with graticules which is made up of radio-opaque markers.

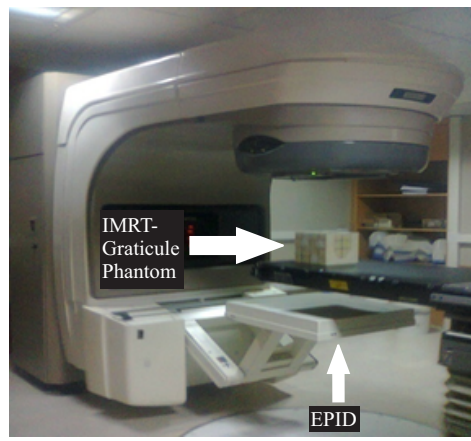

Fig.1. Anterior Setup Image of IMRT Graticule Phantom:-

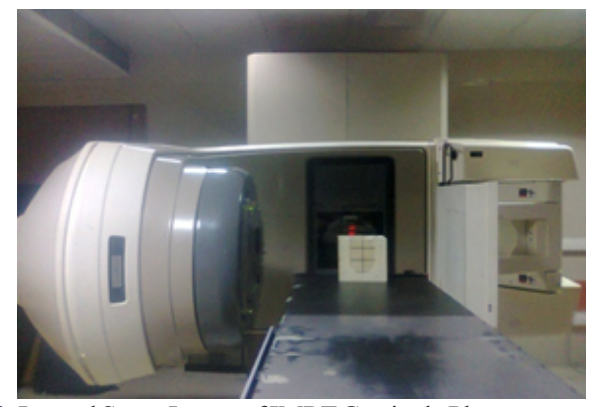

Fig.2. Lateral Setup Image of IMRT Graticule Phantom:-

IMRT Graticule phantom was placed on the couch of CT simulator and three markers made of lead were fixed on the respected faces at the point of intersection of all the three lasers (longitudinal, vertical and horizontal). CT of the phantom was taken on the CT simulator with slice thickness of $2.5 \mathrm{~mm}$. These CT images were sent to the planning system through DICOM (Digital Imaging and communications in medicine). Then a SAD (Source to axis distance) plan was made for Linac (Clinac DBx -1160) and setup field for anterior, left lateral and 
right lateral portal images were added to the existing plan. Digitally Reconstructed Radiograph (DRR) of the phantom was generated for each setup field on the TPS system (Eclipse version 11.6).

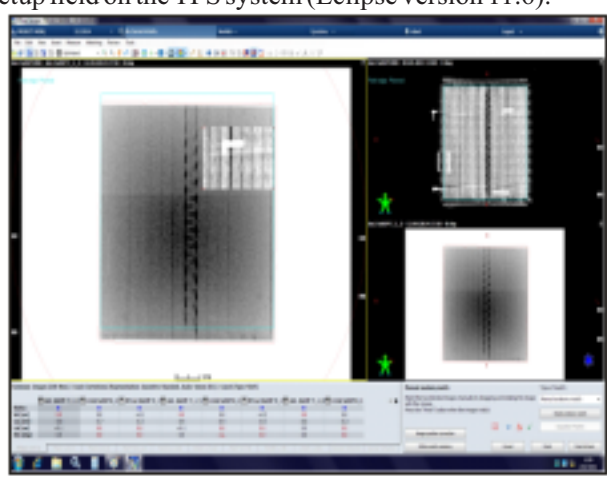

Fig.3. Shows the DRR image at right top position, acquired image from EPID at right bottom position and blended postion on the left side and expected shifts and observed shifts as shown below in table.

- Plan was scheduled on the given Medical Linear Accelerator.

- IMRT phantom was placed on the couch of the machine in a position reproducible as during the time of $\mathrm{CT}$ acquisition. Isocenter shift was applied in all the directions $( \pm \mathrm{x}, \pm \mathrm{y}$ and $\pm \mathrm{z})$ according to the TPS Plan. This position was considered as the original position of the given IMRT Phantom. EPID was taken for Anterior, Left lateral and Right Lateral.

- A known shift of $0.5 \mathrm{~cm}, 1 \mathrm{~cm}$ and $1.5 \mathrm{~cm}$ were introduced in longitudinal (In and Out or $\pm \mathrm{y}$ ), lateral (right and left or $\pm \mathrm{x}$ ) and vertical (up and down or $\pm \mathrm{z}$ ) directions respectively and EPID was taken for each and every shift at a particular source to imager distance (SID).

EPID was repeated for same shift at varying Source to Imager Distance (SID). Source to Imager distance was kept at P2 $=140 \mathrm{~cm}, \mathrm{P} 3=150 \mathrm{~cm}$, $\mathrm{P} 4=160 \mathrm{~cm}$ and $\mathrm{P} 5=180 \mathrm{~cm}$ respectively.

These portal images were matched manually on the console monitor as well as in the offline review of TPS and measured shift were recorded for each portal image as shown in Fig.3.

The difference between expected shifts and observed shifts were found and recorded and standard deviation was calculated as shown in table 1 and 2 below.

Table.1. For Anterior EPID

\begin{tabular}{|c|c|c|c|}
\hline $\begin{array}{c}\text { EPID } \\
\text { Position } \\
\text { (SID) (cm) }\end{array}$ & $\begin{array}{c}\text { Long. } \\
\text { Direction } \\
\text { (Std.Dev.) } \\
\text { (cm) }\end{array}$ & $\begin{array}{c}\text { Lat. Direction } \\
\text { (Std.Dev.) } \\
\text { (cm) }\end{array}$ & $\begin{array}{c}\text { Vert. Direction } \\
\text { (Std.Dev.) } \\
\text { (cm) }\end{array}$ \\
\hline P2 (140) & 0.077 & 0.137 & 0 \\
\hline P3 (150) & 0.133 & 0.079 & 0 \\
\hline P4 (160) & 0.087 & 0.011 & 0 \\
\hline P5 (180) & 0.058 & 0.058 & 0 \\
\hline
\end{tabular}

Table2. For Lateral EPID

\begin{tabular}{|c|c|c|c|}
\hline $\begin{array}{c}\text { EPID } \\
\text { Position } \\
\text { (SID) } \\
\text { (cm) }\end{array}$ & $\begin{array}{c}\text { Long. } \\
\text { Direction } \\
\text { (Std.Dev.) } \\
\text { (cm) }\end{array}$ & $\begin{array}{c}\text { Lat. Direction } \\
\text { (Std.Dev.) } \\
\text { (cm) }\end{array}$ & $\begin{array}{c}\text { Vert. Direction } \\
\text { (Std.Dev.) } \\
\text { (cm) }\end{array}$ \\
\hline P2 (140) & 0.142 & 0 & 0.083 \\
\hline P3 (150) & 0.144 & 0.04 & 0.029 \\
\hline P4 (160) & 0.079 & 0 & 0.092 \\
\hline P5 (180) & 0.014 & 0 & 0.168 \\
\hline
\end{tabular}

RESULTS

From this study it has been found that in anterior Electronic Portal Image the maximum deviation is $0.137 \mathrm{~cm}$ which is at (SID) $140 \mathrm{~cm}$ in lateral direction and minimum deviation is $0.011 \mathrm{~cm}$ which is at (SID) $160 \mathrm{~cm}$ in lateral direction as shown in Fig. 4 and there is no variation found in vertical direction which is our requirement. And for lateral Electronic Portal Image the maximum deviations found is $0.168 \mathrm{~cm}$ at (SID) $180 \mathrm{~cm}$ in vertical direction and minimum deviation is $0.014 \mathrm{~cm}$ at (SID) $180 \mathrm{~cm}$ in longitudinal direction as shown in Fig.5. From the overall study the maximum deviation is less than $2 \mathrm{~mm}$ which is our requirement.

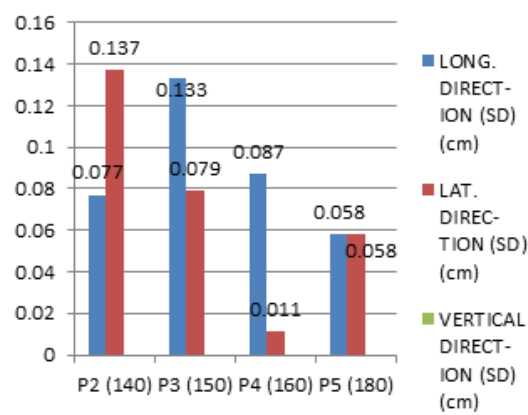

Fig.4. Shows the variation in average standard deviation at different (SID) in anterior EPID in all the directions

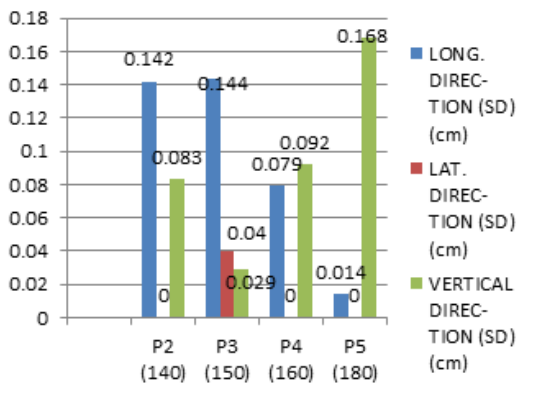

Fig.5. Shows the variation in average standard deviation at different (SID) in lateral EPID in all the directions.

\section{DISCUSSION}

The data in above tables and graphs have shown that in the anterior portal images a maximum average standard deviation of $0.137 \mathrm{~cm}$ at $140 \mathrm{~cm}$ SID in lateral direction and a minimum of $0.011 \mathrm{~cm}$ at SID $160 \mathrm{~cm}$ along the lateral direction and there is no variation in vertical direction which is our requirement and in the lateral portal image recorded a maximum average standard deviation of $0.168 \mathrm{~cm}$ at $180 \mathrm{~cm}$ SID in vertical direction and minimum of $0.01 \mathrm{~cm}$ for $180 \mathrm{~cm}$ SID along the longitudinal direction which is almost negligible. A study conducted by Das et al "A quality assurance phantom for electronic portal imaging device" have studied the appropriate procedure to carry out the Quality Assurance of an EPID. [8] They conduct their studies on PTW EPID QC phantom with associated image analysis software provides a convenient, automated and easy process for periodic verification of performance of EPID with regard to baseline values. Coen.W.Hurkmans et al "Set-up verification using portal imaging; Review of current clinical practice" In this review different causes of set-up errors are summarized. The accuracy of set-up measurements is largely dependent on the availability of software for (automatic) matching of reference images and portal images, inter observer variation for manual matching of images can be very large and are institution dependent. For head and neck treatments, the current 'state of art' set-up accuracy is $2 \mathrm{~mm}$ (1D) standard deviation of random and systematic set-up error, while this is $2.5,3.0$ and $3.5 \mathrm{~mm}$ for prostate, pelvis and lung cancer treatments respectively. For breast and mantle field/total body treatments, larger deviations are found as accuracy with which patients have to be positioned depends on the treatment site and intent to treatment (palliative or curative). For conformal techniques, however, systematic set-up errors of several millimeters standard deviation may already lead to a too high percentage of treatment fractions with unacceptably large 3D set-up errors. Michael G.Herman study on "Clinical use of electronic portal imaging" showed that accurate and routine target localization is necessary for successful outcome in radiation therapy treatments.[9] Electronic portal imaging devices (EPIDs) provide an advanced tool with digital technology to improve target localization and maintain clinical efficiency. Milecki et al carry out the study under the "Evaluation of an electronic portal imaging device (target view, GE) as a quality assurance tool" - a proper control of the geometrical accuracy of treated patients during radiotherapy results in higher quality of treatment and may lead to the increase in therapeutic gain.[10] In this study on EPID positional accuracy with IMRT graticule phantom, the breathing motions are completely neglected due to fixed geometry of phantom. Any variation 
in reference DRR (Digitally Reconstructed Radiograph) and the acquired images are either because of personal error, accuracy of software for automatic matching or inbuilt error of the EPID. Anterior and lateral EPIDs are taken for the intentionally introduced errors $0.5 \mathrm{~cm}, 1.0 \mathrm{~cm}$ and $1.5 \mathrm{~cm}$ in Longitudinal (In and Out or $\pm \mathrm{y}$ ), lateral (Left and Right or $\pm \mathrm{x}$ ) and vertical (Up and Down or $\pm \mathrm{z}$ ) directions for different source to imager distance $(\mathrm{SID})$ i.e. $\mathrm{P} 2=140 \mathrm{~cm}, \mathrm{P} 3=150 \mathrm{~cm}$, $\mathrm{P} 4=160 \mathrm{~cm}$ and $\mathrm{P} 5=180 \mathrm{~cm}$. Average difference and standard deviation of difference (error) are compared at each imager position for anterior and lateral portal images. A maximum standard deviation was reported in at imager position $180 \mathrm{~cm}$ from the source. This is may be because of the following reasons. Imager at this position is very much close to the ground and value of acceleration due to gravity is more as compare to all other imager positions. R-arm of EPID is in maximum extended position. During lateral portals the normal to the surface of EPID is always perpendicular to patient plane. This will leads to sagging in the EPID due to gravity owing to its weight. But the nature of this sagging is quite complex. The two forces which results in sagging are Tension forces along the R-arm of EPID and its weight toward the centre of earth. The value of tension in the EPID is also more as compare to tension in the EPID in other positions of R-arms of EPID. This resultant force will makes the EPID tilt in such a way that the superior side of the EPID will sag towards the couch. Although the angle through which sagging will takes place may be very much small but this leads to a maximum average standard deviation of error as compare to other positions of imager. In the anterior portal, at maximum extended position of imager at $180 \mathrm{~cm}$ the longitudinal sagging takes place. Farthest region of imager in longitudinal direction toward the couch may be move in upward direction towards the gantry. Resultant force due to tension in the R-arm and weight of EPID may be the cause for error in the longitudinal direction. But there is no significant contribution of this error in longitudinal direction. With anterior portal at $150 \mathrm{~cm}$ and $140 \mathrm{~cm}$ SID, shows maximum standard deviation along longitudinal direction and lateral direction respectively. This deviation may be because of non reproducibility of EPID for that instant of taking image or may be due to personnel error while manually matching the reference image and acquired image.

\section{CONCLUSION}

IMRT Graticule phantom can play an important role for the quality assurance of Electronic portal image device (EPID). R-arm of EPID shows a variation in the position reproducibility but it is not so significant. A maximum standard deviation in lateral portal is reported at source to imager distance $180 \mathrm{~cm}$. This is because of sagging owing to Tension force which acts along the R-arm of EPID and its weight toward the centre of earth. Although the angle through which sagging will takes place may be very much small but this leads to a maximum average standard deviation of error as compared to other positions of imager. In the anterior portal, farthest region of imager in longitudinal direction toward the couch may be move in upward direction towards the gantry. Resultant force due to tension in the R-arm and weight of EPID may be the cause for error in the longitudinal direction. Although these errors are not so significant but it is helpful in assessing the inbuilt error in EPID and to make out necessary correction while doing the online patient shift.

\section{REFERENCES}

(1) Hall C. Geometric transformation of portal field edge data: In: Geometric uncertainties in Radiotherapy. London: BIR, 2003:44-45.

(2) Coen W, Hurkmans, Remeijer P et al (2001) Set-up verification using portal imaging; review of current clinical practice. Radiother Oncol 58:105-120

(3) Stroom JC, de Boer HC, Huizenga H, Visser AG. Inclusion of geometrical uncertainities in radiotherapy treatment planning by means of coverage probability. Int J Radiat Oncol Biol Phys 1999; 43(4):905-919.

(4) Alasti H, Petric MP, Catton CN.Warde PR. Portal imaging for evaluation of daily on-line setup errors and off-line organ motion during conformal irradiation of carcinoma of setup errors and off-line organ motion during confor
prostate. Int J Radiat Oncol Biol Phys 2001;49:869-84.

(5) International commission on radiation units and measurements. Prescribing, Recording, and reporting photon beam therapy (Report 50). Bethesda: international commission on radiation units and measurements, 1993.

(6) International commission on radiation units and measurements. Prescribing, Recording, and reporting photon beam therapy Report 62 (supplement to ICRU Report 50). Bethesda: International commission on radiation units and measurements, 1999.

(7) Portal Vision ${ }^{\mathrm{TM}}$ aS500 Rel.6. Reference Manual.

(8) Das et al A Quality Assurance Phantom for Electronic Portal Imaging Device Journal of Applied Clinical Medical Physics, vol.12, Nov.2, spring 2011.

(9) Michael G. Hurkmans et al Clinical Use of Portal Imaging Semin Radat Oncol 15:157$167 \mathrm{c} 2005$.

(10) Milecki et al Evaluation of an electronic portal imaging device (target view,GE) as a quality assurance tool. Reports of Practical Oncology \& Radiotherapy Volume 6, Issue 4, 2001, Pages 169-172. 